\title{
Novel SCN1A variants in Dravet syndrome and evaluating a wide approach of patient selection
}

\author{
Milan Surovy ${ }^{1}$, Andrea Soltysova ${ }^{1,2}$, Miriam Kolnikova $^{3}$, Pavol Sykora $^{3}$, Denisa Ilencikova ${ }^{4}$, \\ Andrej Ficek ${ }^{1}$, Jan Radvanszky ${ }^{1,2}$ and Ludevit Kadasi ${ }^{1,2}$ \\ ${ }^{1}$ Department of Molecular Biology, Faculty of Natural Sciences, Comenius University, Mlynska dolina Bratislava, 84215 \\ Slovak Republic \\ ${ }^{2}$ Center for Molecular Medicine, Slovak Academy of Sciences, Vlarska 7 Bratislava, 83101 Slovak Republic \\ ${ }^{3}$ Department of Child Neurology, Children's Faculty Hospital, Limbova 1 Bratislava, 83340 Slovak Republic \\ ${ }^{4}$ 2nd Pediatric Clinic Faculty of Medicine, Children's University Hospital, Limbova 1 Bratislava, 83340 Slovak Republic
}

\begin{abstract}
Voltage-gated sodium channels are essential for generation and propagation of the action potential mainly in nerve and muscle cells. Causative variants in SCN1A gene which codes the main, pore-forming subunit of the channel expressed in central nervous system are associated predominantly with Dravet syndrome (DS), as well as with generalized epilepsy with febrile seizures plus (GEFS+) making it one of the most significant epilepsy gene. Our goal was to determine whether SCN1A screening is relevant in patients with a broad range of epileptic syndromes. 52 patients diagnosed with DS, GEFS+ or similar types of epileptic syndromes were included. Sequencing of the protein-coding parts of the gene complemented with MLPA analysis was carried out. One already described nonsense variant, four novel protein truncating variants and a deletion encompassing the whole SCN1A gene were revealed, all in heterozygous state. All identified variants were found in DS patients with $85.7 \%$ sensitivity, thus supporting the role of profound SCN1A gene variants in etiology of DS phenotype. No causative variants were identified in any of non-DS epileptic patients in our cohort, suggesting a minor, but not irrelevant role for SCN1A in patients with other types of childhood epilepsy.
\end{abstract}

Key words: Dravet syndrome - Early onset epilepsy - SCN1A - GEFS+

\section{Introduction}

Epilepsies are a clinically and genetically heterogeneous group of neurological disorders characterized by recurrent and unprovoked seizures. One of the most severe epileptic syndromes is Dravet syndrome (DS; OMIM 607208) which is a rare intractable epileptic encephalopathy with onset in infancy (Dravet et al. 2005). Criteria for diagnosis of DS are based on International League Against Epilepsy (ILAE) classification (Berg et al. 2010). This severe condition is often accompanied by various neurological complications and mental decline. Chronic infections, weak humoral immunity

Correspondence to: Milan Surový, Department of Molecular Biology, Faculty of Natural Sciences, Comenius University, Ilkovičova 6, 84215 Bratislava 4, Slovak Republic

E-mail: milan.surovy015@gmail.com and nutrition problems are also common complications. Severity of the disorder is demonstrated by up to $15 \%$ mortality before the age of 20 mostly by sudden unexplained death in epilepsy (Dravet et al. 2005).

Frequent but far less severe genetic generalized epilepsy with febrile seizures-plus (GEFS+; OMIM 604233) is a familial syndrome with autosomal dominant inheritance in which patients suffer from frequent simple febrile seizures in early childhood, from 3 months to 6 years, and later may develop epilepsy characterized by multiple types of seizure, i.e. myoclonic, atonic or partial (Singh et al. 2001; Scheffer et al. 2009).

Since 2001, when SCN1A (sodium channel, voltage gated, type I alpha subunit) mutations were identified to cause DS (Claes et al. 2001), it is the most frequently reported gene associated with epilepsy syndromes, especially DS and other variations of GEFS+ (Escayg et al. 2000; Sugawara et 
al. 2001a; Wallace et al. 2001). Although SCN1A variants are found to be causal in an autosomal dominant manner in approximately $70-80 \%$ of DS patients with more than 800 pathogenic variants identified so far (Claes et al. 2001; Ohmori et al. 2002; Claes et al. 2009), only $10 \%$ of GEFS+ families possess a heterozygous pathogenic SCN1A variant (Scheffer et al. 2009). In addition, severe DS is in the majority of cases associated with profound de novo protein truncating or splice-site SCN1A variants (Claes et al. 2001; Ohmori et al. 2002), while GEFS+ family members transmit mostly moderate missense variants (Escayg et al. 2000; Wallace et al. 2001). As the severity of symptoms generally correlates with the type of the sequence alteration, with more severe phenotypes observed in patients with nonsense or frameshift causing variants, Scheffer et al. (2005) hypothesized, that both the clinically more sever DS and the much milder GEFS+ phenotypes represent just two ends of the same spectrum of SCN1A-related epilepsies.

The aim of the present study was to screen the proteincoding parts of the SCN1A gene for pathogenic variations in patients with a broad range of epileptic phenotypes. Through a thorough phenotypic characterisation and SCN1A findings we evaluate our extensive approach of patient selection and define the most proper genetic testing strategy of patients with epileptic syndromes in clinical practice.

\section{Material and Methods}

During the study, clinical and genetic characterization of 52 unrelated patients presenting characteristic symptoms of childhood epilepsies, including DS (7 patients), LennoxGastaut syndrome (5 patients) and other related syndromes (40 patients) was completed. All patients were clinically diagnosed and classified according to the diagnostic criteria established by the Commission on Classification and terminology of the ILAE (Berg et al. 2010). Only patients with seizure onset before third year of age and with 3 or more clinical signs present -1 . normal development before seizure onset, 2. occurrence of multiple seizure types (myoclonic seizures, atypical absences, partial seizures, afebrile seizures, generalized tonic-clonic or hemiclonic seizures), 3. family history of epilepsy or febrile convulsion, 4 . abnormal EEG findings, 5. resistance to antiepileptic drugs and 6. prolonged convulsive seizures usually triggered by fever or heating - were included in our study. Patients' clinical status and details are summarized in Table 1. Written informed consents consistent to the Helsinki declaration were obtained from parents or legal guardians.

Genomic DNA, from peripheral blood samples collected in potassium EDTA buffered tubes (S-Monovette, Sarsterdt, Germany), was extracted using the Gentra PureGene Blood Kit (Qiagen, Germany) according to the manufacturers recommendations. The whole protein coding sequence of the SCN1A gene was analyzed by standard Sanger sequencing of preamplified exonic regions. Intronic primer pairs to selectively amplify each of the 26 coding exons and exon/intron boundaries of the SCN1A gene (ENSG00000144285) were designed based on the transcript sequence (ENST00000303395) using the Primer3 program (Untergasser et al. 2012) (primer sequences available upon request). Each exon was preamplified using the designed primers and purified by a conventional polyethylene glycol and ethanol precipitation. Amplicons were sequenced in both directions using the BigDye Terminator v3.1 Cycle Sequencing kit chemistry (Applied Biosystems/Life Technologies, USA) and an ABI Prism 3130xl Genetic Analyzer (Applied Biosystems/Life Technologies, USA). Raw data was analyzed using the Sequencing Analysis Software v5.3 with a subsequent alignment to the reference sequence (ENST00000303395) and variant calling through the SeqScape Software v2.5 (Applied Biosystems/Life Technologies, USA). DS patients without identified SCN1A sequence alternations in the coding region and exon/intron boundaries were further analysed with a commercial SCN1A specific Multiplex Ligation-dependent Probe Amplification (MLPA) assay (SALSA P137 MLPA kit; MRC-Holland, The Netherlands) to screen for duplications or deletions of larger regions which are not detectable through conventional Sanger sequencing. MLPA amplicons were separated on an ABI Prism 3130xl Genetic Analyzer (Applied Biosystems/ Life Technologies, USA) and sized using the GeneScan ${ }^{\mathrm{Tm}} 500$ LIZ $^{\circledR}$ Size Standard (Applied Biosystems/Life Technologies, USA). Data analyses were performed using Coffalyser.Net (MRC-Holland, The Netherlands). Identified variants were annotated manually exploiting literature searches, locus specific and general variant database mining; e.g. SCN1A Variant Database (Claes et al. 2009), Human Gene Mutation Database (HGMD) (Stenson et al. 2009), dbSNP (Sherry et al. 2001), as well as by in silico variant effect predictions obtained using Human Splice Finder (Desmet et al. 2009) and MutationT@ster (Schwarz et al. 2014). Final variant interpretation was performed according to the actual "Standards and guidelines for the interpretation of sequence variants" (Richards et al. 2015). Based on either pathogenic criteria with very strong, strong, moderate or supporting evidence of pathogenicity; or benign criteria with stand-alone, strong and supporting evidence of benign impact, sequence variants were defined as pathogenic, likely pathogenic, benign, likely benign or with uncertain significance.

\section{Results}

Sequence analysis of all coding exons and consensus splice sites of the SCN1A gene was performed in 52 unrelated 


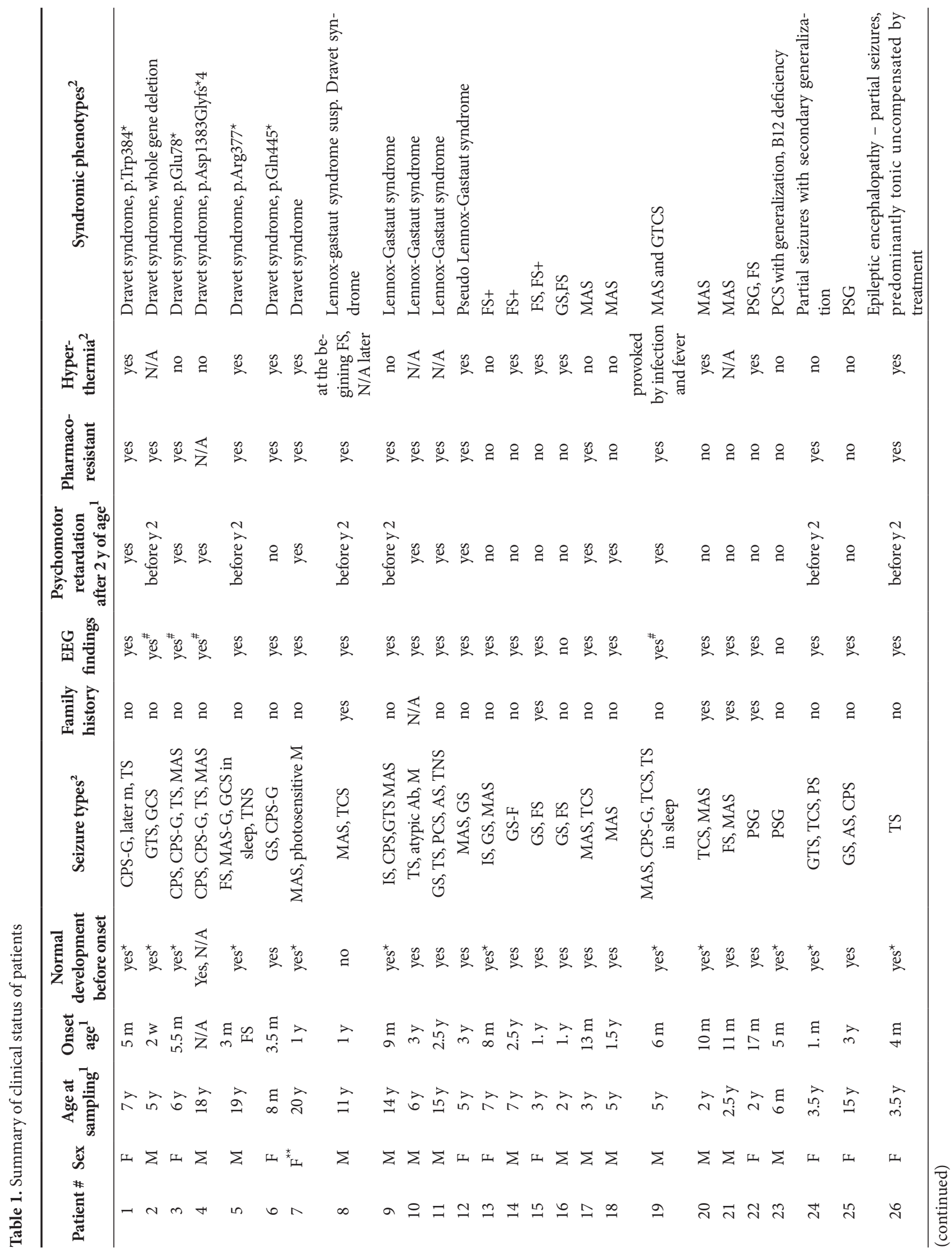




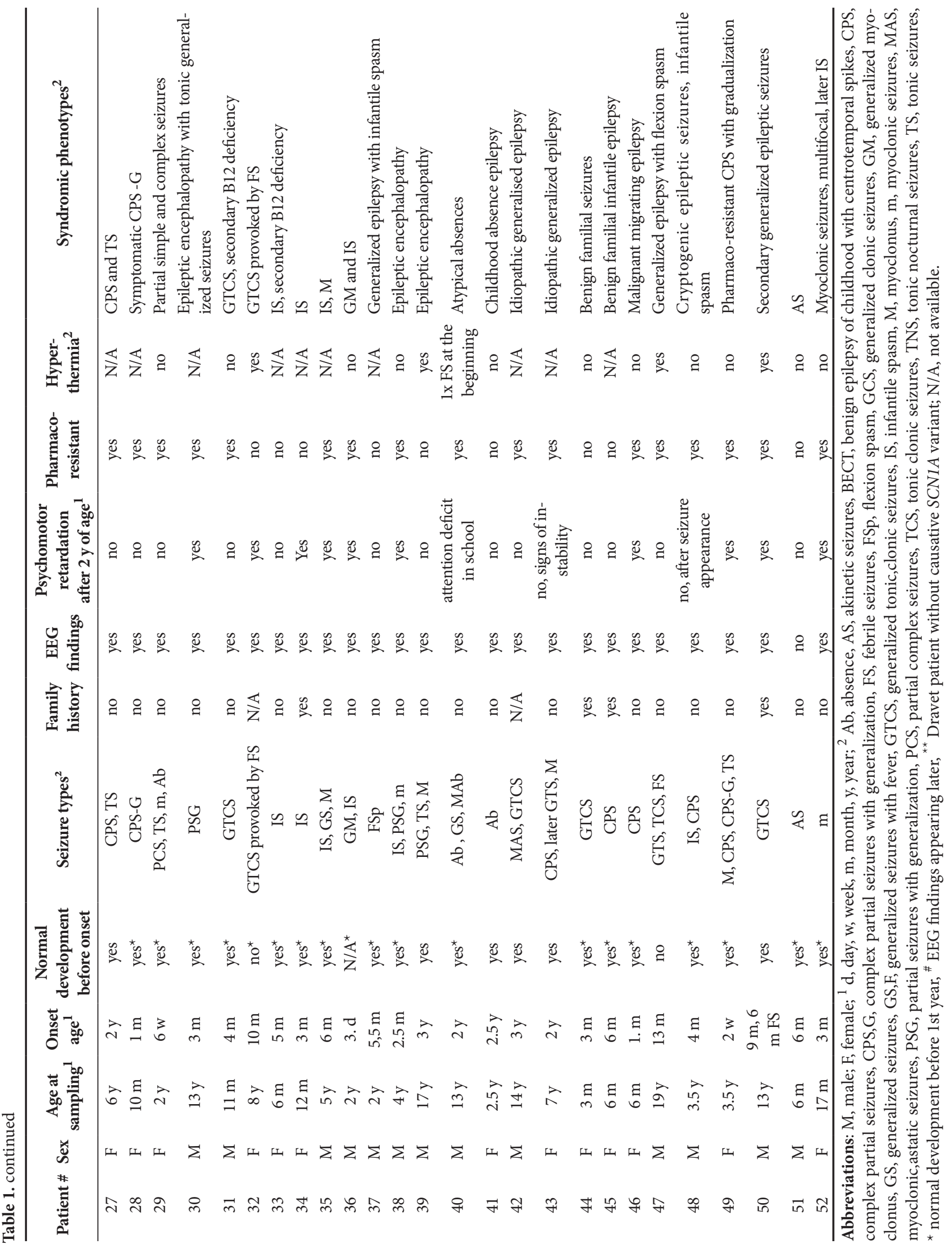




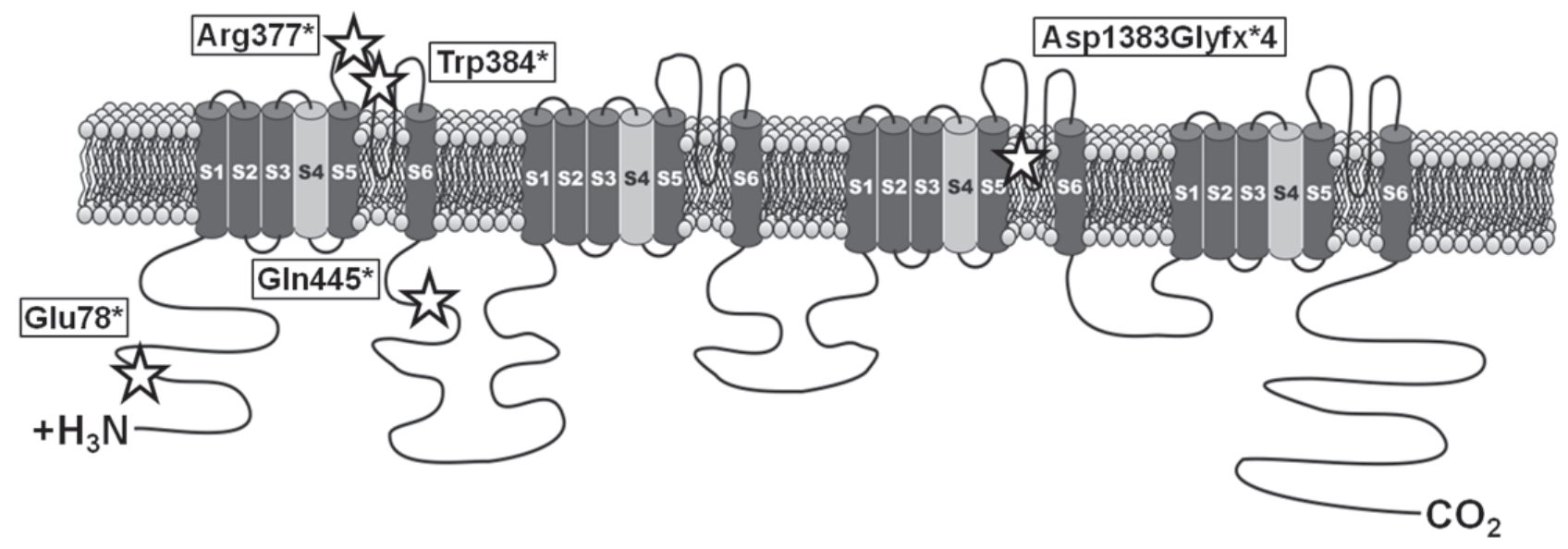

Figure 1. Location of identified likely pathogenic variants within the SCN1A protein.

patients with various types of childhood epilepsy (Table 1). Alignment to the used reference sequence and variant calling revealed altogether 14 different variants. Five of these, all in heterozygous state, represented protein truncating and thus likely pathogenic variants with one very strong and one moderate evidence of pathogenicity. These were identified in patients clinically diagnosed with DS. The remaining 9 variants were identified in the whole sample set and were classified as benign, likely benign or of uncertain significance. During subsequent MLPA analyses a larger deletion encompassing the whole SCN1A gene was identified in one additional DS patient.

\section{Found variants}

From the five different likely pathogenic variants identified in DS patients only one, namely c.1129C > T (p.Arg377*) in exon 8 of the SCN1A gene in patient \#5, was published previously (Depienne et al. 2009). As the remaining variants were not published before, at least according to our best knowledge, we considered them as novel. Nonsense variant c. $232 \mathrm{G}>\mathrm{T}$ (p.Glu78 ${ }^{*}$ ) in first exon is located in the $\mathrm{N}$-terminal region of the SCN1A protein and thus leads to significantly truncated transcript in patient \#3. The third identified variant, c.1151G $>$ A (p.Trp384*) located in exon 8, which codes for S5-S6 D1 domain of the protein, introduces a premature UAG stop codon which, if it would be translated, would lead to the truncation of the nascent protein to only one fifth of the full length in patient \#1. Similarly, the fourth nonsense variant, c.1333C > T (p.Gln445*) in exon 9 in patient \#6, would cause a noticeable shortening of the alpha subunit. We identified also a single base insertion (c.4144-4145insG) located in exon 21 that causes a frameshift introducing a premature stop codon (p.Asp1383Glyfs ${ }^{\star} 4$ ) in the loop between the fifth and sixth transmembrane segment of domain III in patient \#4. Location of each variant within the SCN1A protein is illustrated in Fig. 1.

No pathogenic or likely pathogenic SCN1A variants were found in any of the patients with other types of epilepsy analysed in this study. On the other hand, beside the protein truncating variants, we identified also 9 different SNVs (single nucleotide variant), all summarized in Table 2. According to the dbSNP database and 1000 Genomes Project, frequencies of the minor allele (MAF) for individual SNVs 1-7 lie between 15.6 to 49.8 percent and therefore can be classified as benign with stand-alone evidence of benign impact. In case of SNV 8, based on a low minor allele frequency (MAF = $0.32 \%$ ) we have performed a computational analysis (Desmet et al. 2009; Schwarz et al. 2014) and based on the in silico predictions we assume that this variant is likely benign with supporting evidence of benign impact. SNV 9 is being classified as benign in dbSNP/ClinVar by three submitters, but the evidence is not available to our laboratory to perform an independent evaluation. Moreover, results of two prediction software (Desmet et al. 2009; Schwarz et al. 2014) indicate, that guanine at c.5418 position is fairly evolutionary conserved and alternation may affect splicing. Based on these indications, we have classified SNV 9 as a variant of uncertain significance. Although it should be mentioned, that both of these variants were identified also in homozygous state in 12 (SNV 8) and 33 individuals (SNV 9) in 1000 Genomes Project and Exome Aggregation Consortium.

\section{MLPA analysis}

Since Sanger sequencing of the SCN1A coding region revealed likely pathogenic variants only in five out of seven tested DS patients, MLPA analysis of the SCN1A gene was performed in the remaining two patients. This analysis revealed a heterozygous deletion of the whole SCN1A gene in patient \#2 
Table 2. Summarized benign or likely benign variants

\begin{tabular}{|c|c|c|c|c|c|c|c|}
\hline \multirow{2}{*}{ SNV\# } & \multicolumn{2}{|c|}{ Variant } & \multirow{2}{*}{ RS\# } & \multirow{2}{*}{$\begin{array}{l}\text { Clinical Significance } \\
\text { (dbSNP/ClinVar) }\end{array}$} & \multirow{2}{*}{ MAF } & \multirow{2}{*}{$\begin{array}{c}\text { MAF } \\
\text { (this study) }\end{array}$} & \multirow{2}{*}{$\begin{array}{c}\text { Location in } \\
\text { SCN1A }\end{array}$} \\
\hline & cDNA & Protein & & & & & \\
\hline 1. & c.965-21C>T & - & rs994399 & N/A & $C=0.2909$ & $C=0.5961$ & intron 6 \\
\hline 2. & c. $1028+21 \mathrm{~T}>\mathrm{C}$ & - & rs 1542484 & N/A & $\mathrm{C}=0.4980$ & $C=0.5865$ & intron 7 \\
\hline 3. & c. $1212 \mathrm{~A}>\mathrm{G}$ & $\mathrm{Val}=$ & rs7580482 & other & $A=0.2907$ & $A=0.2980$ & exon 9 \\
\hline 4. & c. $1663-47 \mathrm{G}>\mathrm{T}$ & - & rs6753355 & N/A & $\mathrm{T}=0.2107$ & $\mathrm{~T}=0.3076$ & intron 10 \\
\hline 5. & c. $2292 \mathrm{~T}>\mathrm{C}$ & $\mathrm{Val}=$ & rs 6432860 & With Benign, other allele & $\mathrm{T}=0.2111$ & $\mathrm{~T}=0.3461$ & exon 13 \\
\hline 6. & c. $2416-72 \mathrm{G}>\mathrm{A}$ & - & rs490317 & N/A & $A=0.1560$ & $A=0.0096$ & intron 13 \\
\hline 7. & c. $3199 \mathrm{G}>\mathrm{A}$ & Ala $>$ Thr & rs2298771 & other & $\mathrm{G}=0.2116$ & $\mathrm{G}=0.5192$ & exon 16 \\
\hline 8. & c. $3705+33 \mathrm{~T}>\mathrm{G}$ & - & rs76743139 & N/A & $\mathrm{G}=0.0032$ & $\mathrm{G}=0.0096$ & intron 18 \\
\hline 9. & c. $5418 \mathrm{G}>\mathrm{A}$ & $\mathrm{Glu}=$ & rs140237315 & With Benign allele & $A=0.0094$ & $A=0.0096$ & exon 26 \\
\hline
\end{tabular}

Clinical significance was determined according to dbSNP database (Sherry et al. 2001); N/A, not available.

while we still did not identify any possibly causative variant in one patient with DS (indicated as " "* in Table 1). MLPA analysis wasn't performed in patients that didn't show features of severe DS phenotype, since it seems to be unlikely that deletions/duplications of the SCN1A gene would be compatible with milder forms of epilepsy (Marini et al. 2009).

\section{Discussion}

Although in the present study we screened in total of $52 \mathrm{pa}-$ tients with a wide range of epileptic syndromes, only seven of them were clinically diagnosed to have severe DS. Likely pathogenic SCN1A variants were identified exclusively in six of seven patients, while, with respect to the protein functionality, these variations belonged to the most severe types. All but one would lead to early termination of translation and thus to significantly C-truncated SCN1A proteins if the transcripts would not enter the nonsense-mediated decay pathway. The only exception was the deletion of the whole SCN1A gene that leads to a complete loss of one copy of the gene supporting the hypothesis that SCN1A haploinsufficiency may be the major cause of symptoms also in DS patients with early truncating mutations. It should be mentioned, however, that in contrast to other DS patients, carrier of this deletion (patient \#2) was diagnosed at a much younger age (2 weeks) then the others (3.5-5.5 months). As we mentioned above, from the presently reported variants only the c.1129C>T (p.Arg377*) variant in exon 8 (Depienne et al. 2009) and different whole gene deletions (Sherry et al. 2001; Stenson et al. 2009) were previously reported in connection to DS. One of the remaining four variants, p.Trp $384^{*}$ that is in our case caused by a c.1151G $>$ A substitution changing a TGG codon to TAG was, however, previously described as a result of the adjacent c.1152G $>$ A transition leading to a TGG to TGA codon change (Harkin et al. 2007).

Unfortunately, since we had no parental DNA samples available we were not able to conclusively prove by segregation analyses neither the pathogenic potential of the novel variants nor their de novo origin in our patients. These can be, however, presumed with a relatively high probability as it is generally accepted that haploinsufficiency or gain of toxic function of truncated proteins may play a crucial role in the pathogenesis of the disease. Loss-of-function SCN1A sequence variants, such as nonsense and frameshift causing ones, were reported to be the predominant genetic abnormalities seen in DS patients and are usually associated with the most severe phenotypes and reproductive disadvantage (Sugawara et al. 2001; Marini et al. 2007). Most of the already described truncating variants, similarly to those identified by us, are found in the $5^{\prime}$ end of the SCN1A gene while the proteins altered in such way likely undergoes a nonsensemediated decay (NMD) pathway (Holbrook et al. 2004). Ohmori et al. (2006) for example, demonstrated that almost all SCN1A truncating variants associated with DS markedly reduce inward sodium currents and thus causes loss of function of the SCN1A protein. Further studies revealed that loss-of-function variants found in DS patients and studied in mouse models induce hyperexcitability and decrease of $\mathrm{Na}^{+}$current in neocortical (Ogiwara et al. 2007) and hippocampal (Yu et al. 2006) $\gamma$-aminobutyric acid (GABA) ergic inhibitory interneurons. Volker and colleagues, on the other hand, performed and described a biophysical analysis of an identified variant, which surprisingly causes DS by overall gain of function (Volkers et al. 2011). It should also be mentioned that in some cases missense variants in SCN1A can also be associated with DS (Ohmori et al. 2002; Fukuma et al. 2004); however, we did not identify possibly pathogenic 
missense variations in DS patients screened in the study. When considering only clinically diagnosed DS cases, combination of sequencing and MLPA testing approach showed a $85.7 \%$ sensitivity of causative DNA sequence variant detection, even higher than was reported in previous studies: 82.7\% (Ohmori et al. 2002), 71\% (Marini et al. 2007), 73\% (Depienne et al. 2009). The higher sensitivity observed in present study could be the result of very strict criteria during clinical diagnosis. Based on the hypothesis posed by Scheffer et al. that DS and GEFS+ are just two ends of the same spectrum of SCN1A-related epilepsies one can imagine that in the clinical practice patients with milder phenotypes can also represent patients with functionally milder SCN1A mutations or even patients with yet not fully manifested DS symptoms (Scheffer et al. 2005). This hypothesis seems to be supported by previously reported frequencies of SCN1A causative variants in patients with GEFS+ which lies between 3-10\% (Escayg et al. 2001; Wallace et al. 2001; Ceulemans et al. 2004; Scheffer et al. 2009). Although SCN1A causative variants do not explain the majority of GEFS+ cases this does not mean that their contribution is clinically unimportant. However, our findings seem to show, that SCN1A is not the most relevant causal gene in the pathogenesis of milder phenotypes belonging to the GEFS+ spectrum and to other types of epilepsy, since mutation screening of the gene in 45 non-DS patients did not reveal any pathogenic or at least possibly pathogenic variant. Common clinical feature for all non-DS patients enrolled in our study was a normal development before seizure onset (42/45), EEG findings (42/45) and resistance to multiple anticonvulsants (25/45). It is also possible that $S C N 1 A$ causative variants were not found simply due to a small number of patients enrolled in our study. Besides that, variants in a broad group of genes, for example gamma-aminobutyric acid (GABA)A receptor g2 subunit gene (Harkin et al. 2002), SCN2A (Kamiya et al. 2004; Shi et al. 2009; Feenstra et al. 2014), SCN9A (Singh et al. 2009), PCDH19 (Depienne et al. 2009), and another candidate gene, SCN1B (Patino et al. 2009), may be considered responsible in epileptic phenotypes at all. Very similar set of candidate genes were shown to be responsible also for GEFS+, i.e. SCN1A, GABRG2, SCN2A, SCN1B and GABRD (Wallace et al. 1998; Baulac et al. 2001; Sugawara et al. 2001; Audenaert et al. 2003; Dibbens et al. 2004), although the SCN1A ones are more frequent than variants in the other listed GEFS+ genes altogether. Thus patients, who are found to not harbour pathogenic SCN1A variants, can be tested for variations in the above mentioned genes.

It is widely accepted for clinically highly heterogeneous phenotypes, that the mutation detection rate is often closely linked to the way of the assessment of differential diagnosis as well as to the selection of proper DNA testing algorithm. Various approaches and diagnostic criteria, all with their pros and cons, can be used during clinical evaluation of pa- tients with epileptic symptoms prior to genetic testing to minimize genetic heterogeneity among patients. However, studies using a wide approach in patient selection with various epileptic phenotypes are also used. In one such study by Fountain-Capal et al. a mutation detection rate of $23 \%$ was observed in a group of 69 epileptic/DS children patients (Fountain-Capal et al. 2011). Authors mentioned that a relatively open clinical testing strategy was used in their clinic for patient selection. According to their data, a sensitivity of $94 \%$ and a specificity of about $60 \%$ would be achieved using additional exclusive criteria such as either exacerbation of symptoms with hyperthermia or normal development before the onset of seizures prior to the mutation testing in children that show symptoms associated with DS. Using this approach, the number of tested children would be reduced by approximately $50 \%$, on the other hand approximately $5 \%$ of cases may be overlooked. The appearance of ataxia, pyramidal signs, or interictal myoclonus alone has not been shown to be determining for an effective guidance in testing, however, Bremer and co-workers believe that DS may be even overlooked in many patients with pharmaco-resistant seizures and learning disability, particularly in the adult population (Bremer et al. 2012). Based on our observation we may agree with Marini and assume that detected discrepancy in detection rate can be attributed to the controversial aspects in the diagnosis of DS, as it can be uncertain, especially in younger patients (Marini et al. 2007). Definition of borderline phenotypes of the DS spectrum may be even more problematic, as some crucial features, such as psychomotor regression, EEG changes, seizure progression, presence or absence of ataxia, may occur after prolonged phase of clinical observation. Even though modified criteria from those proposed by the ILAE (2001) were used in most of the studies, some authors propose a clinical scoring system for screening at high-risk patients for genetic testing of DS including seven clinical predictive risk factors, i.e. onset within the first 7 months of life, more than five seizures, hemiconvulsions, focal seizures, myoclonic seizures, prolonged seizures, and hot water-induced seizures (Hattori et al. 2008).

In the light of several findings discussed above, our findings seem to prove that both proper patient selection criteria in research settings as well as appropriate genetic testing strategy in clinical practice are very crucial parts of the overall workflow, therefore should be evidence-based and performed cautiously. This is important not only for Sanger sequencing based, gene-by-gene testing approach, but also in newer testing strategies based on massively parallel sequencing. Ongoing transition of genomic analyses from research into the routine clinical practice raised serious debates on appropriate testing strategies with several emerging guidelines for developing standards for best practices. Concerns have been raised, for example, because of the question of unsolicited and secondary findings. Therefore several guidelines 
suggest careful selection of genes to be included in targeted gene panel approaches or careful bioinformatic filtration of only those variants which are found in genes clearly associated with the patient's symptoms in case of exome or whole genome approaches (Rehm et al. 2013; Matthijs et al. 2014). On the other hand, using relatively loose diagnostic criteria, that may seems to be unnecessary and even costly in following genetic tests, may minimize the probability of preclinical cases to be overlooked. Caraballo et al. suggested that the possibility of DS should be considered in patients exhibiting febrile seizures (FS) as DS is characterized by the occurrence of FS before the first year of age, however, these are often diagnosed only at the age of 2-4 years following the manifestation of distinctive clinical features. Thus patients with repeating and prolonged FS triggered by fever, often misdiagnosed as benign FS in its early stages, should be treated as DS patients (Caraballo and Fejerman 2006).

In clinically highly heterogeneous groups of disorders with overlapping symptomatology genetic testing may provide early and accurate diagnosis that can be used to support a suspicion for a certain clinical entity even in the first stages of the disease. Definitive diagnosis in case of positive DNA screening can therefore enhance treatment with appropriate anticonvulsants and temperature reducing drugs selection and ultimately saves time. Although SCN1A is the most relevant epilepsy gene with hundreds of pathogenic variants identified so far, these are found mainly in patients suffering from the most severe phenotypes. Moreover it should be also taken to consideration that criteria for clinical differential diagnosis of DS patients may be more or less variable during selection of patients for genetic screening studies. We therefore propose to screen children with severe symptoms, in which it is likely that they will develop DS, although there's a risk that these patients have a different epileptic disorder. It would be a good compromise, as early treatment is crucial and can't be put on hold until all characteristic DS symptoms arise.

Acknowledgements. This contribution is the result of the project implementation: Comenius University in Bratislava Science Park supported by the Research and Development Operational Program funded by the ERDF. Grant number: ITMS 26240220086 and by Slovak Grant Agency of Science (VEGA 1/1288/12 and $1 / 0236 / 13)$.

Conflict of interest. None of the authors has any conflict of interest to disclose. We confirm that we have read the Journal's position on issues involved in ethical publication and affirm that this report is consistent with those guidelines.

\section{References}

Audenaert D., Claes L., Ceulemans B., Löfgren A., Van Broeckhoven C., De Jonghe P. (2003): A deletion in SCN1B is associated with febrile seizures and early-onset absence epilepsy. Neurology 61, 854-856 http://dx.doi.org/10.1212/01.WNL.0000080362.55784.1C

Baulac S., Huberfeld G., Gourfinkel-An I., Mitropoulou G., Beranger A., Prud'homme J. F., Baulac M., Brice A., Bruzzone R., LeGuern E. (2001): First genetic evidence of GABA(A) receptor dysfunction in epilepsy: a mutation in the gamma2-subunit gene. Nat. Genet. 28, 46-48 http://dx.doi.org/10.1038/ng0501-46

Berg A. T., Berkovic S. F., Brodie M. J., Buchhalter J., Cross J. H., van Emde Boas W., Engel J., French J., Glauser T. A., Mathern G. W. et al. (2010): Revised terminology and concepts for organization of seizures and epilepsies: report of the ILAE Commission on Classification and Terminology, 2005-2009. Epilepsia 51, 676-685

http://dx.doi.org/10.1111/j.1528-1167.2010.02522.x

Bremer A., Lossius M. I., Nakken K. O. (2012): Dravet syndrome-considerable delay in making the diagnosis. Acta. Neurol. Scand. 125, 359-362 http://dx.doi.org/10.1111/j.1600-0404.2011.01609.x

Caraballo R. H., Fejerman N. (2006): Dravet syndrome: a study of 53 patients. Epilepsy Res. 70, 231-238

http://dx.doi.org/10.1016/j.eplepsyres.2005.11.026

Ceulemans B. P., Claes L. R., Lagae L. G. (2004): Clinical correlations of mutations in the SCN1A gene: from febrile seizures to severe myoclonic epilepsy in infancy. Pediatr. Neurol. 30, 236-243

http://dx.doi.org/10.1016/j.pediatrneurol.2003.10.012

Claes L., Del-Favero J., Ceulemans B., Lagae L., Van Broeckhoven C., De Jonghe P. (2001): De novo mutations in the sodiumchannel gene SCN1A cause severe myoclonic epilepsy of infancy. Am. J. Hum. Genet. 68, 1327-1332 http://dx.doi.org/10.1086/320609

Claes L. R., Deprez L., Suls A., Baets J., Smets K., Van Dyck T., Deconinck T., Jordanova A., De Jonghe P. (2009): The SCN1A variant database: a novel research and diagnostic tool. Hum. Mutat. 30, 904-920

http://dx.doi.org/10.1002/humu.21083

Depienne C., Trouillard O., Saint-Martin C., Gourfinkel-An I., Bouteiller D., Carpentier W., Keren B., Abert B., Gautier A., Baulac S. et al. (2009): Spectrum of SCN1A gene mutations associated with Dravet syndrome: analysis of 333 patients. J. Med. Genet. 46, 183-191

http://dx.doi.org/10.1136/jmg.2008.062323

Desmet F. O., Hamroun D., Lalande M., Collod-Béroud G., Claustres M., Béroud C. (2009): Human Splicing Finder: an online bioinformatics tool to predict splicing signals. Nucleic Acid Res. 37, e67 http://dx.doi.org/10.1093/nar/gkp215

Dibbens L. M., Feng H. J., Richards M. C., Harkin L. A., Hodgson B. L., Scott D., Jenkins M., Petrou S., Sutherland G. R., Scheffer I. E. et al. (2004): GABRD encoding a protein for extra- or peri-synaptic GABAA receptors is a susceptibility locus for generalized epilepsies. Hum. Mol. Genet. 13, 1315-1319 http://dx.doi.org/10.1093/hmg/ddh146

Dravet C., Bureau M., Oguni H., Fukuyama Y., Cokar O. (2005): Severe myoclonic epilepsy in infancy: Dravet syndrome. Adv. Neurol. 95, 71-102 
Escayg A., MacDonald B. T., Meisler M. H., Baulac S., Huberfeld G., An-Gourfinkel I., Brice A., LeGuern E., Moulard B., Chaigne D. (2000): Mutations of SCN1A, encoding a neuronal sodium channel, in two families with GEFS+2. Nat. Genet. 24, 343-345 http://dx.doi.org/10.1038/74159

Escayg A., Heils A., MacDonald B. T., Haug K., Sander T., Meisler M. H. (2001): A novel SCN1A mutation associated with generalized epilepsy with febrile seizures plus--and prevalence of variants in patients with epilepsy. Am. J. Hum. Genet. 68, 866-873 http://dx.doi.org/10.1086/319524

Feenstra B., Pasternak B., Geller F., Carstensen L., Wang T., Huang F., Eitson J. L., Hollegaard M. V., Svanström H., Vestergaard M. et al. (2014): Common variants associated with general and MMR vaccine-related febrile seizures. Nat. Genet. 46, 1274-1282 http://dx.doi.org/10.1038/ng.3129

Fountain-Capal J. K., Holland K. D., Gilbert D. L., Hallinan B. E. (2011): When should clinicians order genetic testing for Dravet syndrome? Pediatr. Neurol. 45, 319-323 http://dx.doi.org/10.1016/j.pediatrneurol.2011.08.001

Fukuma G., Oguni H., Shirasaka Y., Watanabe K., Miyajima T., Yasumoto S., Ohfu M., Inoue T., Watanachai A., Kira R. et al. (2004): Mutations of neuronal voltage-gated $\mathrm{Na}+$ channel alpha 1 subunit gene SCN1A in core severe myoclonic epilepsy in infancy (SMEI) and in borderline SMEI (SMEB). Epilepsia 45, 140-148 http://dx.doi.org/10.1111/j.0013-9580.2004.15103.x

Harkin L. A., Bowser D. N., Dibbens L. M., Singh R., Phillips F., Wallace R. H., Richards M. C., Williams D. A., Mulley J. C., Berkovic S. F. et al. (2002): Truncation of the GABA(A)receptor gamma2 subunit in a family with generalized epilepsy with febrile seizures plus. Am. J. Hum. Genet. 70, 530-536 http://dx.doi.org/10.1086/338710

Harkin L. A., McMahon J. M., Iona X., Dibbens L., Pelekanos J. T., Zuberi S. M., Sadleir L. G., Andermann E., Gill D., Farrell K. et al. (2007): The spectrum of SCN1A related infantile epileptic encephalopathies. Brain 130, 843-852 http://dx.doi.org/10.1093/brain/awm002

Hattori J., Ouchida M., Ono J., Miyake S., Maniwa S., Mimaki N., Ohtsuka Y., Ohmori I. (2008): A screening test for the prediction of Dravet syndrome before one year of age. Epilepsia 49, 626-633 http://dx.doi.org/10.1111/j.1528-1167.2007.01475.x

Holbrook J. A., Neu-Yilik G., Hentze M. W., Kulozik A. E. (2004): Nonsense-mediated decay approaches the clinic. Nat. Gene. 36, 801-808 http://dx.doi.org/10.1038/ng1403

Kamiya K., Kaneda M., Sugawara T., Mazaki E., Okamura N., Montal M., Makita N., Tanaka M., Fukushima K., Fujiwara T. et al. (2004): A nonsense mutation of the sodium channel gene SCN2A in a patient with intractable epilepsy and mental decline. J. Neurosci. 24, 2690-2698 http://dx.doi.org/10.1523/JNEUROSCI.3089-03.2004

Marini C., Mei D., Temudo T., Ferrari A. R., Buti D., Dravet C., Dias A. I., Moreira A., Calado E., Seri S. et al. (2007): Idiopathic epilepsies with seizures precipitated by fever and SCN1A abnormalities. Epilepsia 48, 1678-1685

http://dx.doi.org/10.1111/j.1528-1167.2007.01122.x

Marini C., Scheffer I. E., Nabbout R., Mei D., Cox K., Dibbens L. M., McMahon J. M., Iona X., Carpintero R. S., Elia M. et al. (2009): SCN1A duplications and deletions detected in Dravet syndrome: implications for molecular diagnosis. Epilepsia 50, $1670-1678$ http://dx.doi.org/10.1111/j.1528-1167.2009.02013.x

Matthijs G. (2014): Guidelines for diagnostic next generation sequencing. Final draft version ES_2-12-2014 (https://www.eshg. org/fileadmin/www.eshg.org/documents/EGT/EuroGentest_ NGS_guidelines_2014_-_final_draft_02-12-2014_v2.pdf)

Ogiwara I., Miyamoto H., Morita N., Atapour N., Mazaki E., Inoue I., Takeuchi T., Itohara S., Yanagawa Y., Obata K. et al. (2007): Nav1.1 localizes to axons of parvalbumin-positive inhibitory interneurons: a circuit basis for epileptic seizures in mice carrying an Scn1a gene mutation. J. Neurosci. 27, 5903-5914

http://dx.doi.org/10.1523/JNEUROSCI.5270-06.2007

Ohmori I., Ouchida M., Ohtsuka Y., Oka E., Shimizu K. (2002): Significant correlation of the SCN1A mutations and severe myoclonic epilepsy in infancy. Biochem. Biophys. Res. Commun. 295, 17-23

http://dx.doi.org/10.1016/S0006-291X(02)00617-4

Ohmori I., Kahlig K. M., Rhodes T. H., Wang D. W., George A. L. Jr. (2006): Nonfunctional SCN1A is common in severe myoclonic epilepsy of infancy. Epilepsia 47, 1636-1642

http://dx.doi.org/10.1111/j.1528-1167.2006.00643.x

Patino G. A., Claes L. R., Lopez-Santiago L. F., Slat E. A., Dondeti R. S., Chen C., O'Malley H. A., Gray C. B., Miyazaki H., Nukina N., Oyama F. et al. (2009): A functional null mutation of SCN1B in a patient with Dravet syndrome. J. Neurosci. 29, 10764-10778 http://dx.doi.org/10.1523/JNEUROSCI.2475-09.2009

Rehm H. L., Bale S. J., Bayrak-Toydemir P., Berg J. S., Brown K. K., Deignan J. L., Friez M. J., Funke B. H., Hegde M. R., Lyon E.; Working Group of the American College of Medical Genetics and Genomics Laboratory Quality Assurance Commitee. (2013): ACMG clinical laboratory standards for next-generation sequencing. Genet. Med. 15, 733-747 http://dx.doi.org/10.1038/gim.2013.92

Richards S., Aziz N., Bale S., Bick D., Das S., Gastier-Foster J., Grody W. W., Hegde M., Lyon E., Spector E. et al.; ACMG Laboratory Quality Assurance Committee. (2015): Standards and guidelines for the interpretation of sequence variants: a joint consensus recommendation of the American College of Medical Genetics and Genomics and the Association for Molecular Pathology. Genet. Med. 17, 405-423 http://dx.doi.org/10.1038/gim.2015.30

Sherry S. T., Ward M. H., Kholodov M., Baker J., Phan L., Smigielski E. M., Sirotkin K. (2001): dbSNP: the NCBI database of genetic variation. Nucleic Acids Res. 29, 308-311 http://dx.doi.org/10.1093/nar/29.1.308

Shi X., Yasumoto S., Nakagawa E., Fukasawa T., Uchiya S., Hirose S. (2009): Missense mutation of the sodium channel gene SCN2A causes Dravet syndrome. Brain Dev. 31, 758-762 http://dx.doi.org/10.1016/j.braindev.2009.08.009 
Scheffer I. E., Harkin L. A., Dibbens L. M., Mulley J. C., Berkovic S. F. (2005): Neonatal epilepsy syndromes and generalized epilepsy with febrile seizures plus (GEFS+). Epilepsia 46 Suppl. $10,41-47$ http://dx.doi.org/10.1111/j.1528-1167.2005.00358.x

Scheffer I. E., Zhang Y. H., Jansen F. E., Dibbens L. (2009): Dravet syndrome or genetic (generalized) epilepsy with febrile seizures plus? Brain Dev. 31, 394-400 http://dx.doi.org/10.1016/j.braindev.2009.01.001

Schwarz J. M., Cooper D. N., Schuelke M., Seelow D. (2014): MutationTaster2: mutation prediction for the deep-sequencing age. Nat. Methods. 11, 361-362 http://dx.doi.org/10.1038/nmeth.2890

Singh N. A., Pappas C., Dahle E. J., Claes L. R., Pruess T. H., De Jonghe P., Thompson J., Dixon M., Gurnett C., Peiffer A. et al. (2009): A role of SCN9A in human epilepsies, as a cause of febrile seizures and as a potential modifier of Dravet syndrome. PLoS Genet 5, e1000649 http://dx.doi.org/10.1371/journal.pgen.1000649

Singh R., Andermann E., Whitehouse W. P., Harvey A. S., Keene D. L., Seni M. H., Crossland K. M., Andermann F., Berkovic S. F., Scheffer I. E. (2001): Severe myoclonic epilepsy of infancy: extended spectrum of GEFS+? Epilepsia 42, 837-844 http://dx.doi.org/10.1046/j.1528-1157.2001.042007837.x

Stenson P. D., Mort M., Ball E. V., Howells K., Phillips A. D., Thomas N. S., Cooper D. N. (2009): The Human Gene Mutation Database: 2008 update. Genome Med. 1, 13 http://dx.doi.org/10.1186/gm13

Sugawara T., Mazaki-Miyazaki E., Ito M., Nagafuji H., Fukuma G., Mitsudome A., Wada K., Kaneko S., Hirose S., Yamakawa K. (2001a): Nav1.1 mutations cause febrile seizures associated with afebrile partial seizures. Neurology 57, 703-705 http://dx.doi.org/10.1212/WNL.57.4.703

Sugawara T., Tsurubuchi Y., Agarwala K. L., Ito M., Fukuma G., Mazaki-Miyazaki E., Nagafuji H., Noda M., Imoto K., Wada K. et al. (2001b): A missense mutation of the $\mathrm{Na}+$ channel alpha
II subunit gene $\mathrm{Na}(\mathrm{v}) 1.2$ in a patient with febrile and afebrile seizures causes channel dysfunction. Proc. Natl. Acad. Sci. USA 98, 6384-6389 http://dx.doi.org/10.1073/pnas.111065098

Untergasser A., Cutcutache I., Koressaar T., Ye J., Faircloth B. C., Remm M., Rozen S. G. (2012): Primer3--new capabilities and interfaces. Nucleic Acids Res. 40, 115 http://dx.doi.org/10.1093/nar/gks596

Volkers L., Kahlig K. M., Verbeek N. E., Das J. H., van Kempen M. J., Stroink H., Augustijn P., van Nieuwenhuizen O., Lindhout D., George A. L. Jr. et al. (2011): Nav 1.1 dysfunction in genetic epilepsy with febrile seizures-plus or Dravet syndrome. Eur. J. Neurosci. 34, 1268-1275 http://dx.doi.org/10.1111/j.1460-9568.2011.07826.x

Wallace R. H., Wang D. W., Singh R., Scheffer I. E., George A. L. Jr., Phillips H. A., Saar K., Reis A., Johnson E. W., Sutherland G. R. et al. (1998): Febrile seizures and generalized epilepsy associated with a mutation in the $\mathrm{Na}+$-channel betal subunit gene SCN1B. Nat. Genet. 19, 366-370 http://dx.doi.org/10.1038/448

Wallace R. H., Scheffer I. E., Barnett S., Richards M., Dibbens L., Desai R. R., Lerman-Sagie T., Lev D., Mazarib A., Brand N. et al. (2001): neuronal sodium-channel $\alpha 1$-subunit mutations in generalized epilepsy with febrile seizures plus. Am. J. Hum. Genet. 68, 859-865 http://dx.doi.org/10.1086/319516

Yu F. H., Mantegazza M., Westenbroek R. E., Robbins C. A., Kalume F., Burton K. A., Spain W. J., McKnight G. S., Scheuer T., Catterall W. A. (2006): Reduced sodium current in GABAergic interneurons in a mouse model of severe myoclonic epilepsy in infancy. Nat. Neurosci. 9, 1142-1149

http://dx.doi.org/10.1038/nn1754

Received: October 13, 2015

Final version accepted: January 18, 2016

First published online: April 5, 2016 